\title{
Bericht vom Forschungssymposium Physiotherapie an der Universität Lübeck und von der 3. Mitgliederversammlung der DGPTW
}

Am 16. und 17. November 2018 begrüßten die Universität zu Lübeck und die Deutsche Gesellschaft für Physiotherapiewissenschaft (DGPTW) die wissenschaftlich tätigen und an Wissenschaft interessierten Physiotherapeuten auf dem Campus der Universität zu Lübeck ( $\triangleright$ Abb. 1). Bei schönstem Wetter trafen sich im hohen Norden rund 300 Teilnehmende aus ganz Deutschland, der Schweiz und Österreich. Das Symposium empfahl sich erneut als eine ausgezeichnete Plattform, auf der sich Vertreter aller physiotherapierelevanten Forschungsbereiche präsentieren und austauschen konnten. Die thematischen Schwerpunkte lagen in der muskuloskeletalen und klinischen Forschung, Neurorehabilitation, Methodenforschung und der Darstellung der Physiotherapie im Wandel.

Der 1. Symposiumstag begann mit den Treffen der Sektionen der DGPTW. Seit deren Gründung hat sich die Anzahl der Experten in den jeweiligen Sektionen „Öffentlichkeitsarbeit“, „Leitlinien“, „Methoden/Beratung“, „Kongress“ und „Theorieentwicklung in der Physiotherapie“ erfreulicherweise weiter erhöht. In ungezwungenem Rahmen diskutierten die Sektionen ihre Schwerpunkte und nahmen viele neue Ideen und Aufgaben mit ins nächste Jahr.

Im weiteren Vorprogramm des Symposiums fanden die Workshops statt, in denen wissenschaftlich tätige Physiotherapeuten über ihre aktuellen Forschungsfelder referierten. Je nach Interessenslage konnten die Teilnehmenden im Vorfeld 2 von insgesamt 6 Workshops auswählen. Viele der Workshops stießen bereits im Vorfeld auf so großes Interesse, dass sie ausgebucht waren. Die Themen deckten wieder eine weite, interessante Bandbreite ab und wurden sowohl praxisorientiert als auch evidenzbasiert vorgestellt. Die Workshops reichten von der Bedeutung der OutcomeForschung in der Physiotherapie (Christian Kopkow) bis hin zu praktischen, validen Mobilitätsmessinstrumenten bei älteren Patienten, welche leicht in den klinischen Alltag integriert werden können (Tobias

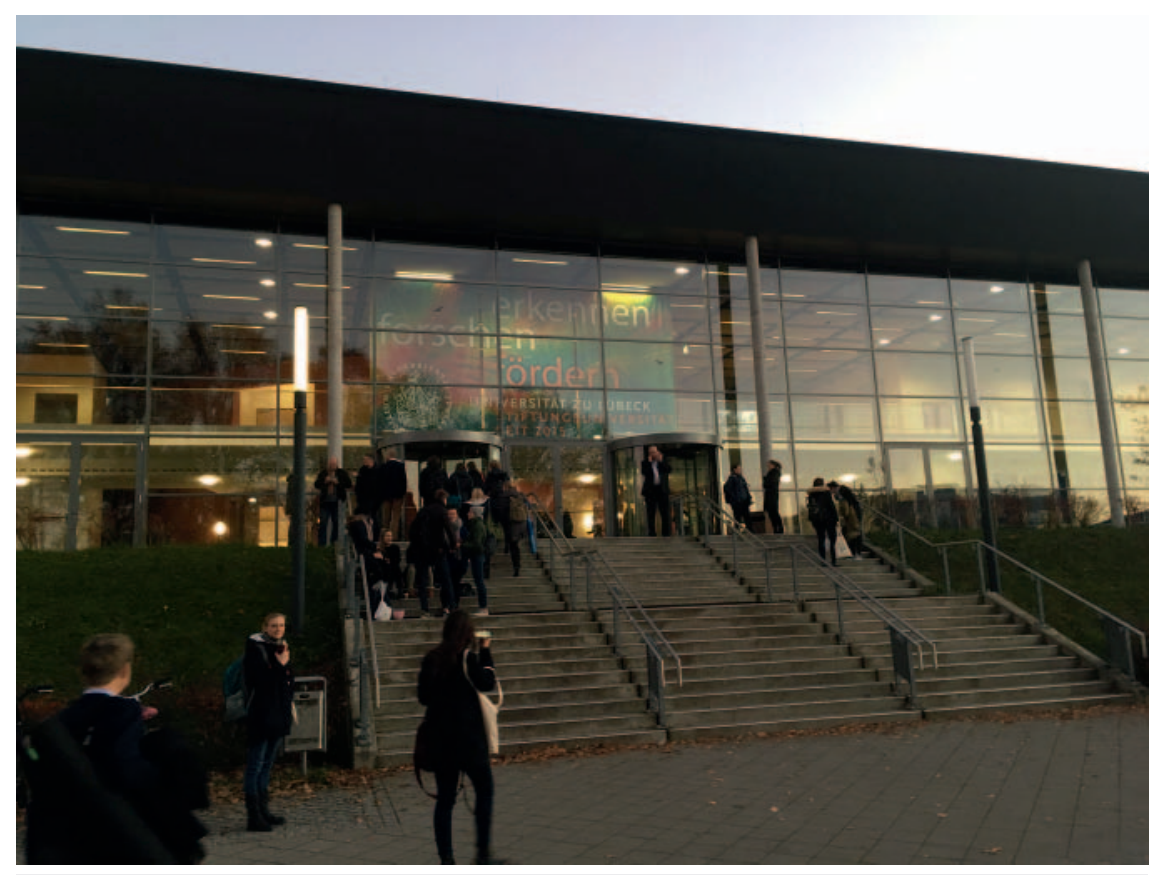

- Abb.1 Campus der Universität zu Lübeck. (Quelle: M. Gronwald)

Braun). In einem weiteren Workshop setzte sich Tibor Szikszay mit der endogenen Schmerzmodulation bei chronischen Schmerzen und ihrem Nutzen für Physiotherapeuten auseinander. Minettchen Herchenröder und Annett Heitling gaben ein Update zur Ganganalyse. Das Programm rundeten die Workshops zur Erstellung von Cochrane Reviews (Bernhard Elsner) und zu Möglichkeiten der virtuellen Realität als Intervention für die Neurorehabilitation (Nina Rohrbach) ab.

\section{Mitgliederversammlung der DGPTW}

Nach einer kurzen Stärkung stand die 3. Mitgliederversammlung der DGPTW (www.dgptw.org) an ( $\triangleright$ Abb.2). Das Interesse an der DGPTW und ihrer Arbeit wächst erfreulicherweise seit der 1. Mitgliederversammlung in Bochum und der 2. Mitgliederversammlung in Osnabrück weiter: rund 45 ordentliche und assoziierte Mitglieder sowie zahlreiche weitere Interessierte erschienen zur Versammlung. Die DGPTW umfasst aktuell ca.
140 Mitglieder und hat in den sozialen Medien auf Facebook und Twitter sowie ganz neu auch auf Instagram eine große Community.

Nach der Begrüßung durch die 5 Vorstandsmitglieder Kerstin Lüdtke, Axel Schäfer, Bernhard Elsner, Christian Kopkow und Cordula Braun und der damit verbundenen Vorstellung der 5 Sektionen entlasteten die ordentlichen Mitglieder der DGPTW den Vorstand für das Jahr 2017. Nach einem kurzen Bericht über die Entwicklung und den aktuellen Stand der DGPTW stellte Axel Schäfer die Ziele, Visionen und das Leitbild der Gesellschaft stellvertretend für den gesamten Vorstand vor [1]. Die DGPTW begreift sich als eine unabhängige wissenschaftliche Fachgesellschaft, deren Ziele in der Förderung von Wissenschaft und Forschung in der Physiotherapie sowie in der Verknüpfung dieser Wissenschaft und Forschung mit der Lehre und Versorgung liegen. 


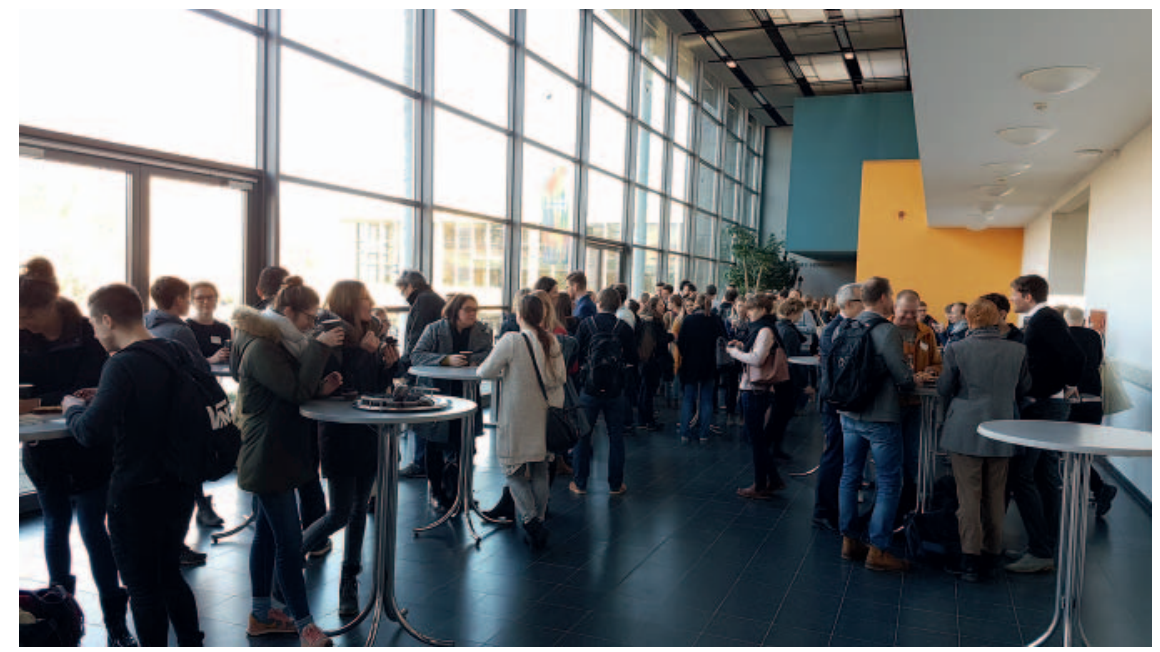

- Abb.2 In den Pausen bestand Gelegenheit zum Netzwerken. (Quelle: M. Gronwald)

\section{Keynote-Vortrag über die Herausforderungen evidenz- basierter Gesundheits- forschung}

Nach der Eröffnung des Symposiums durch die 1. Vorsitzende Kerstin Lüdtke begann das wissenschaftliche Programm mit einem herausragenden Keynote-Redner: Für die DGPTW war es eine besondere Ehre, dass sie Prof. Dr. Gerd Antes (z. Zt. Center for Advanced Studies, CAS, der LMU München) für die Eröffnungs-Keynote gewinnen konnte. Prof. Antes ist den meisten durch seinen herausragenden Einsatz für die evidenzbasierte Medizin und ihrer Umsetzung und Verbreitung in Deutschland sowie in seiner Rolle als Gründer und langjähriger Direktor von Cochrane Deutschland in Freiburg bekannt. In seinem Vortrag erläuterte er unter anderem eindrücklich und anschaulich die großen, auch international viel diskutierten Probleme bei der Generierung, Disseminierung und Implementierung von wissenschaftlicher Evidenz und die sich daraus ergebenden Herausforderungen für die Evidenzbasierung und evidenzbasierte Gesundheitsversorgung, denen sich auch die Physiotherapie stellen muss. Ein in Teilen sehr ernüchternder und nachdenklich stimmender, aber für die Weiterentwicklung der Physiotherapie(-wissenschaft) umso wichtigerer Einblick. Viel Anerkennung zollte Prof. Dr. Antes dem großen Engagement deutscher Physiotherapeuten für die Disseminierung von (Cochrane-)Evidenz im deutschsprachigen Raum, für das er die Physiotherapie in Deutschland in einer Pionier- und Vorzeigerrolle sieht. Er ermutigte ausdrücklich zum „Weitermachen" auf dem bisher eingeschlagenen Weg.

Mit diesem eindrücklichen Plädoyer ging es sichtlich bewegt in die Abendveranstaltung im „The Newport“ über. Bei einem tollen, lokal-kulinarischen Büffet und Wein aus der hauseigenen Winzerei wurde noch lange über die Erlebnisse und Eindrücke des Tages diskutiert.

\section{Placebo- und Noceboeffekte gezielt einsetzen}

Viel Zeit zur Besichtigung der schönen Hansestadt Lübeck blieb auch am nächsten Tag nicht. Schon früh begrüßten der Initiator des Studiengangs Physiotherapie an der Universität zu Lübeck Prof. Dr. Arndt-Peter Schulz und Studiengangsleiterin Prof. Dr. Kerstin Lüdtke die Anwesenden und hießen den 2. Keynote-Redner Dr. Björn Horing vom Institut für systemische Neurowissenschaften am UKE in Hamburg herzlich willkommen. Dr. Horing gab einen Einblick in die Placeboforschung und deren Erkenntnisse, Möglichkeiten und Grenzen in der Grundlagenforschung und im klinischen Alltag. Mit viel rhetorischer Stilsicherheit führte er die Teilnehmenden durch die Geschichte der Placeboforschung bis hin zu deren aktuellen Erkenntnissen. Das vielleicht für manche trocken erscheinende Thema präsentierte er mithilfe sehr vieler praxisrelevanter Beispiele sehr anschaulich. Anhand zahlreicher Studienergebnisse empfahl er den Physiotherapeuten, die Placeboeffekte als Teil ihrer Behandlung anzusehen und Patienten über Placebo- und Noceboeffekte so zu informieren, dass Behandlungseffekte maximiert und Nebenwirkungen minimiert werden können. Hierzu verwies er auf eine gute, empathisch-vertrauensvolle Kommunikation, die bereits in der Ausbildung erlernt werden sollte.

Die folgenden wissenschaftlichen Vorträge waren in die Bereiche „Becken und untere Extremität“, „Physiotherapie im Wandel“, „Wirbelsäule“ und „Parkinson, Schlaganfall und Querschnitt“ eingeteilt und fanden zum Teil parallel in 2 Vortragssälen statt. Dabei war zu beobachten, dass die Vorträge von Bachelor- und MasterAbsolventen problemlos neben den Vorträgen von langjährig tätigen Akademikern bestehen konnten.

Im Anschluss vergab die DGPTW 3 von den Teilnehmenden ausgewählte Vortragspreise. Den 1. Preis erhielt Gesine Seeber für den Vortrag: „The Effects of Posterior Tibial Mobilization on Meniscal Movement: An In-Situ Investigation“, den 2. Preis Katja Ehrenbrusthoff für den Vortrag „Förderfaktoren und Barrieren in der Umsetzung von evidenzbasierter Praxis in der Physiotherapie in Deutschland - vorläufige Ergebnisse einer deutschlandweiten Online-Befragung“ und der 3. Preis ging an Pauline Kuithan für den Vortrag: „Patienten mit Kreuzschmerzen erreichen keine bewegungsinduzierte Hypoalgesie durch eine wiederholte Hebeübung“.

Einen weiteren Preis gab es für das beste Poster. Zum 1. Mal fanden auf der hervorragend bestückten Posterausstellung geführte Posterpräsentationen statt. Der diesjährige, vom Thieme-Verlag vergebene Preis (Buchgutschein über 150 Euro) ging an Sarah Klamroth für ihr Posters „Individuelle Therapieeffekte: Analyse interindividueller Unterschiede in der Anpassung der Gleichgewichtsfähigkeit nach Laufbandtherapie bei Parkinsonpatienten“. Insgesamt waren durch ein Peer-Review-Verfahren 50 Poster und 13 Vorträge ausgewählt worden. 
An dieser Stelle nochmals herzlichen Glückwunsch an alle Gewinnerinnen und vielen Dank an alle Beitragenden für ihre qualitativ hochwertige wissenschaftliche Leistung sowie die überaus positive Atmosphäre auf dem Forschungssymposium in Lübeck.

An den steigenden Teilnehmerzahlen, den vielen eingereichten Abstracts und den positiven Rückmeldungen werden der Stellenwert und die Wichtigkeit eines Forschungssymposiums in der Physiotherapie immer mehr erkennbar. Mit der DGPTW als unabhängige wissenschaftliche Fachgesellschaft erscheint es wichtiger denn je, die junge und hoch motivierte Forschungslandschaft der Physiotherapie in Deutsch- land zu stärken. In gespannter Vorfreude erwarten wir daher das nächste FSPT, das am 22. und 23. November 2019 an der Hochschule für angewandte Wissenschaft und Kunst in Hildesheim stattfindet. Informationen zum Programm gibt es bereits unter www.dgptw.org/forschungssymposium2019-ankuendigung.

Die DGPTW dankt allen Personen, welche für den reibungslosen Ablauf und die großartige Verpflegung auf dem Symposium 2018 sorgten.

Über ihre Aktivitäten berichtet die DGPTW regelmäßig über ihre Social-Media-Kanäle auf Facebook, Instagram und Twitter.
Autorinnen/Autoren

Marit Gronwald

Sektion Öffentlichkeitsarbeit und der Vorstand der DGPTW

marit.gronwald@googlemail.com

\section{Literatur}

[1] Vorstand der Deutschen Gesellschaft für Physiotherapiewissenschaft (DGPTW). Das Leitbild der Deutschen Gesellschaft für Physiotherapiewissenschaft (DGPTW): Vision, Mission und Werte. physioscience 2018; 14 194-196

\section{Bibliografie}

DOI https://doi.org/10.1055/a-0832-9492 physioscience 2019; 15: 45-47

(c) Georg Thieme Verlag KG, Stuttgart · New York ISSN 1860-3092 